\title{
Medicinal Plant Use and Trade Among the Samburu People in Maralal Town, Kenya
}

Lucy Wamuyu Muithui ( $\nabla$ wamuyuwamuyu@gmail.com)

Department of Environmental Studies, Karatina University

\section{Research}

Keywords: Kenya, Medicinal plants, Traditional medicine, Market surveys, Samburu

Posted Date: April 27th, 2021

DOI: https://doi.org/10.21203/rs.3.rs-429123/v1

License: @ (i) This work is licensed under a Creative Commons Attribution 4.0 International License. Read Full License 


\section{Abstract}

Background: The Samburu people have for the longest time used traditional knowledge on medicinal plants to treat diseases both in human and livestock due to the attributed belief of how effective they are. I set out to understand medicinal plant use and trade among the Samburu community in Maralal town. The objectives of this study were to; (1) identify and document the medicinal plants traded, (2) record the medicinal uses of identified plants, (3) document the parts of the plant used and methods of preparation.

Method: I conducted market surveys and held in-depth discussions with the traders on what plant parts were used and sold and where they collected and sourced their plants. I also joined collectors in the wild and collected samples for further identification.

Results: From the market surveys, a total of 24 plants species from 16 families were traded. Most of the plants were collected from Kirisia Forests Reserve and the arid shrublands within Samburu County. It was noted that Myrsine africana L., Rotheca myricoides (Hochst.) Steane \& Mabb., Rhamnus staddo A.Rich., Salvadora persica L. and Toddalia asiatica (L.) Lam were the most popular species with buyers. Most of the plants were used to treat multiple ailments. Stomach related problems and respiratory disorders were some of the commonly treated ailments.

Conclusion: It is evident that use and trade of medicinal plant is an important economic activity in Maralal, however; overharvesting plants from the wild has contributed to some species like Osyris lanceolata Hochst. \& Steud (East African sandalwood) and M. africana becoming rare and scarce hence the need to establish conservation measures and sustainable exploitation.

\section{Introduction}

Medicinal plants are an important ecosystem service in the provision of health care. Traditional knowledge on use of medicinal plants is actively practiced by many around the world. In Africa, communities use medicinal plants for prevention or treatment of diseases in both humans and livestock. To date, traditional knowledge on medicinal plants has traditional healers in sub-Saharan as key providers of healthcare (1) with about $80 \%$ of the population depending on it (2). Even with the availability of medical facilities such as hospitals, health centers, and clinics, the low cost of medicinal plants, its effectiveness, and availability have made it a preferred option especially by the rural population in Kenya (1). Ineffectiveness and dissatisfaction with conventional medicine have made the use of medicinal plants popular (3) especially in treating chronic diseases such as high blood pressure, diabetes, cancer, and hypertension (4). Others use medicinal plants to complement conventional medicine $(5,6)$. In many African countries trade in medicinal plants form part of the informal economy contributing to the livelihood of the traders especially women (7-10). Interesting enough, when it comes to ethnobiological market studies very few or none at all have been carried out in the different parts of the world considering how simple it is (11). It is therefore important to study medicinal plants traded in local markets within a country to document and quantify traded products (12). This can provide estimates of species richness, highlight traded species that require urgent conservation action and especially those that have been overharvested (13). Traditional knowledge on medicinal plants not only shows a country's medicinal flora (8) but highlights the significance of traditional medicine on healthcare (14). Kenya has over 40 ethnic tribes each with its own unique culture and belief. Therefore, the knowledge and use of medicinal plants is different among the tribes. Most of the traditional knowledge on medicinal plants is orally passed down from one generation to next generation within the different tribes. Unfortunately, this important knowledge is quickly disappearing because most of the young generation now prefer white collars jobs in urban cities as alternative sources of income. In all the major urban markets in Kenya, there are traders selling or/and giving advice on medicinal plants products both as raw plant parts or/and readily available dosage that they have prepared themselves (15). Most of these medicinal products are traded as bitter liquid concoctions, bitter powders, sun dried roots, leaves, flowers and fruits. Quantity and quality controls are also questionable because most of the plant products are sold in open air markets where they are openly displayed and exposed to dust particles, they are stored and packed in recycled sacks, newspapers, plastic bottles, tins or bags which may affect the safety and hygiene of the medicine. Also, diagnosis concerns and uncertainty regarding dosage are some of the challenges associated with medicinal plants. In Kenya, there is no regulatory framework on medicinal plant use and trade, no detailed database on medicinal plants with most of the trade being informal and unregulated. There's also a lack of conservation effort of medicinal plants considering majority of the plant products are harvested from the wild. To recognize plant species that require conservation priorities, it is important to understand species traded and amounts sold (14) hence the need for market surveys. The objectives of this study were to; (1) identify and document the medicinal plants traded in Maralal town, (2) record the medicinal uses of identified plants, (3) document the parts of the plant used and methods of preparation.

\section{Material And Methods}

\subsection{Study area}

This study was carried out in Maralal town, Samburu County. It is one of the counties in the Northern drylands of Kenya with an estimated population of 310,327 (16). The county is about $21,000 \mathrm{~km}^{2}$ of which 3,288 square $\mathrm{Km}\left(15.5 \%\right.$ of the County land area) is under gazette forests, $170 \mathrm{Km}^{2}$ is under game reserves and a sanctuary while $1.8 \mathrm{Km}^{2}$ is under surface water. Maralal town $1^{\circ} 5^{\prime} 10.9680^{\prime \prime} \mathrm{N}$ and $36^{\circ} 42^{\prime} 1.2384^{\prime \prime}$ E.(Fig. 1) which is $350 \mathrm{~km}$ from Nairobi is the administrative capital of Samburu county. The town settles at the foothills of Kirisia Hills. The Samburu people form the majority of the population, however, there are other notable communities such as Turkana, Borana, and Pokot. Economic activities revolve around livestock keeping mainly nomadic pastoralists who keep cattle, sheep, goats and camels.

\subsection{Medicinal plant use by the Samburu}

The Samburu are livestock keeping pastoralists living in Northern Kenya. They mainly keep cattle, goats, sheep and sometimes camels, and donkeys. Plant resources play an essential part in life for the Samburu by providing fodder for livestock, construction materials, food cultural practices and traditional 
medicine. Like their relatives (Maasai) in Southern Kenya, The Samburu have for the longest time used medicinal plants that have been attributed by the belief of how effective traditional medicine is (17). Several other studies have documented the same (18-22)

\subsection{The medicinal plant traders}

A total of 33 traders were interviewed. Out of the 33 middle aged traders, only two were men. $90 \%$ of the traders were above 50 years old with the oldest being 68 years. The traders have collectors who supply them with the plants. Majority of the traders are from the Samburu ethnic group and a few from Turkana.

Apart from medicinal plants, the traders also sold other items such as traditional Samburu knives and bead jewelry.

\subsection{Market Surveys and plant collection}

I conducted the market surveys in August 2019 and January 2020 in Maralal town. I purchased medicinal plants from the traders to be conversant with the most used and traded plant species. Traders were also interviewed on a voluntary basis. The interviews were conducted in Samburu language through a translator with a focus on species of plants traded, where the species were collected, cost of the plant product and mode of administration. Information on individual plant products sold, plant parts, uses, and preparation was also recorded. All traded plant parts were identified using local names (Samburu) which were later matched with the Latin names from literature. Most of the plant parts were sold as a single species or in a mixed liquid concoction used to treat specific ailment. The Plant List 2013 (http://www.theplantlist.org/) was used to spell all the Latin names. To further identify and confirm the medicinal uses of the traded plants, review of literature was done. I accompanied three collectors and collected twenty-two specimen samples of the traded plant apart from Osyris lanceolata Hochst. \& Steud and "Lolpiyayo". The plants were collected from the dry shrublands in Maralal, Wamba, and from Kirisia forest and deposited at the Herbarium, National Museums of Kenya. Due to legal restrictions and scarcity of $O$. lanceolata it was impossible to collect any samples. For "Lolpiyayo" none of the available collectors could identify the plant in the wild.

[1] http://www.kenya-information-guide.com/samburu-county.html

\section{Results}

\subsection{Medicinal plants trade and use}

From the market surveys, 24 plant species from 16 families were traded ( Table 1). 15 of the 24 plants were collected from Kirisia Forest Reserve (Table 2), while the other nine were collected from the arid shrublands near Maralal and Wamba towns. Five species were popular with buyers according to the traders Myrsine africana L. (Seketet), Rotheca myricoides (Hochst.) Steane \& Mabb. (Lmakutikuti), Rhamnus staddo A.Rich. (Lkukulai), Salvadora persica L. (Sekotei) and Toddalia asiatica (L.) Lam. (Leparmunyo). Apart from its medicinal values, S. persica was popular for dental cleanliness as a toothbrush. Most of the plants were used to treat more than one ailment. Those used to treat sexually transmitted diseases such as $R$. myricoides were listed as the strongest and most dangerous. Four of the traded plants Ajuga remota Benth, Zanthoxylum chalybeum Engl., Cissus rotundifolia Vahl and Cissus quadrangularis $L$ were also used to treat diseases in livestock. Osyris lanceolata Hochst. \& Steud was one of the traded species and due to overharvesting, it has become rare to find in the wild. The species is currently listed as illegal to harvest or trade any part, in Kenya, however, the traders didn't seem to know this. To portion the quantity of product to sell, a 100 grams recycled tin was used to measure seeds, small pieces of bark, and roots (Picture 1). The price was standard at $\$ 0.45$ per 100 grams for most of the traded parts. A bundle of barks or stems ( Picture 2 and Picture 3 ) was sold at $\$ 1$. Most of the medicinal plants traded were in dry form mainly roots, barks, leaves, and seeds. Also, a cold bitter decoction of eight plant species mixed and boiled together was sold by a few traders. The decoction was known by the local name as "Lekeek isiet" and was made using R. prinoides, T. asiatica, R. staddo, R. myricoides, M. africana, U. massaica, C. spinarum and Croton megalocarpus Hutch. 
Table 1

Medicinal plant species traded in Maralal town: Latin and local names, parts used, treatment, documented uses and pharmacology activities.

\begin{tabular}{|c|c|c|c|c|c|c|c|c|}
\hline $\begin{array}{l}\text { Voucher } \\
\text { Number }\end{array}$ & Family & Latin name & Local name & $\begin{array}{l}\text { Part } \\
\text { used }\end{array}$ & Treatment & $\begin{array}{l}\text { Documented } \\
\text { traditional } \\
\text { uses }\end{array}$ & $\begin{array}{l}\text { Reported } \\
\text { pharmacological } \\
\text { activity }\end{array}$ & Habit \\
\hline MSM20/24 & Anacardiaceae & Rhus natalensis & Lmisigiyoi & $\begin{array}{l}\text { Root, } \\
\text { Bark }\end{array}$ & $\begin{array}{l}\text { Boiled } \\
\text { decoction is } \\
\text { used to treat } \\
\text { cold and flu } \\
\text { in children }\end{array}$ & $\begin{array}{l}\text { Treat } \\
\text { respiratory } \\
\text { disorders and } \\
\text { Malaria (5) }\end{array}$ & $\begin{array}{l}\text { Antibacterial and } \\
\text { antifungal } \\
\text { compounds } \\
\text { from root back } \\
\text { (24). }\end{array}$ & Tree \\
\hline MSM19/10 & Apocynaceae & Carissa spinarum L. & Lamuria & Root & $\begin{array}{l}\text { Root } \\
\text { decoction } \\
\text { treats } \\
\text { arthritis, } \\
\text { rheumatism }\end{array}$ & $\begin{array}{l}\text { Treats } \\
\text { theileriosis, } \\
\text { helminthosis, } \\
\text { rheumatism, } \\
\text { malaria } \\
\text { tuberculosis, } \\
\text { Salmonellosis } \\
\text { (19) STDs, } \\
\text { epilepsy, } \\
\text { malaria, } \\
\text { arthritis (25) }\end{array}$ & $\begin{array}{l}\text { Antioxidant } \\
\text { activity } \\
\text { (Mundaragi and } \\
\text { Thangadurai, } \\
\text { 2018) }\end{array}$ & Shrub \\
\hline MSM20/21 & Canellaceae & Warburgia ugandensis Sprague & Sokoni & Bark & $\begin{array}{l}\text { Bark } \\
\text { decoction } \\
\text { treats } \\
\text { pneumonia, } \\
\text { chest pains }\end{array}$ & $\begin{array}{l}\text { Treats chest } \\
\text { pains (17) }\end{array}$ & $\begin{array}{l}\text { Stem bark } \\
\text { extracts show } \\
\text { antibacterial } \\
\text { activity ( } 27)\end{array}$ & Tree \\
\hline MSM19/15 & Capparaceae & Maerua endlichii Gilg \& Bened & $\begin{array}{l}\text { Lnyiriman } \\
\text { Longososi }\end{array}$ & $\begin{array}{l}\text { Root, } \\
\text { Bark }\end{array}$ & $\begin{array}{l}\text { Bark } \\
\text { decoction for } \\
\text { cleaning the } \\
\text { stomach } \\
\text { through } \\
\text { diarrhoea }\end{array}$ & $\begin{array}{l}\text { Treatment } \\
\text { agains } \\
\text { nematodes in } \\
\text { sheep }(28) \text {, }\end{array}$ & $\begin{array}{l}\text { No reported } \\
\text { activity }\end{array}$ & Shrub \\
\hline MSM20/16 & Ebenaceae & Euclea divinorum Hiern & Lchingei & Bark & $\begin{array}{l}\text { Bark } \\
\text { decoction } \\
\text { treats } \\
\text { malaria, } \\
\text { constipation, } \\
\text { stomachache }\end{array}$ & $\begin{array}{l}\text { Pounded and } \\
\text { applied as an } \\
\text { antivenom } \\
\text { (29) }\end{array}$ & $\begin{array}{l}\text { Antifungal and } \\
\text { antioxidant } \\
\text { activity (30), } \\
\text { antinociceptive } \\
\text { effects (31) }\end{array}$ & Shrub \\
\hline MSM19/6 & Euphorbiaceae & Croton dichogamus $\mathrm{Pax}$ & Lakirding'ai & $\begin{array}{l}\text { Root, } \\
\text { Bark, }\end{array}$ & $\begin{array}{l}\text { Decoction } \\
\text { used for } \\
\text { common } \\
\text { cold, cough }\end{array}$ & $\begin{array}{l}\text { Headaches } \\
(32)\end{array}$ & $\begin{array}{l}\text { Anti- } \\
\text { inflammatory, } \\
\text { antimalarial or } \\
\text { anti-plasmodial } \\
\text { Activities (33) }\end{array}$ & Shrub \\
\hline MSM20/22 & & $\begin{array}{l}\text { Eurphoria candelabrum } \\
\text { Trémaux ex Kotschy }\end{array}$ & Mpopong'i & Leaves & $\begin{array}{l}\text { Decoction is } \\
\text { used to treat } \\
\text { chest pains; it } \\
\text { also cleans } \\
\text { the stomach } \\
\text { through } \\
\text { diarrhea }\end{array}$ & $\begin{array}{l}\text { Treats STIs } \\
\text { and wounds } \\
\text { (19), treats } \\
\text { warts and } \\
\text { tuberculosis } \\
\text { (34) }\end{array}$ & $\begin{array}{l}\text { No reported } \\
\text { activity }\end{array}$ & Tree \\
\hline MSM19/9 & Lamiaceae & Ajuga integrifolia Buch.-Ham & Lmenang'i & $\begin{array}{l}\text { Whole } \\
\text { plant }\end{array}$ & $\begin{array}{l}\text { Decoction in } \\
\text { soup for } \\
\text { stomachache, } \\
\text { malaria, } \\
\text { stops } \\
\text { diarrhoea }\end{array}$ & $\begin{array}{l}\text { Malaria, boils } \\
\text { (35) }\end{array}$ & $\begin{array}{l}\text { Antiplasmodial } \\
\text { properties }(36) \\
(36,37)\end{array}$ & Herb \\
\hline MSM19/1 & & $\begin{array}{l}\text { Rotheca myricoides (Hochst.) } \\
\text { Steane \& Mabb. }\end{array}$ & Lmakutikuti & Root & $\begin{array}{l}\text { Root } \\
\text { decoction } \\
\text { treats chronic } \\
\text { STDs, } \\
\text { pneumonia, } \\
\text { malaria, } \\
\text { fertility in } \\
\text { women }\end{array}$ & $\begin{array}{l}\text { Malaria, } \\
\text { asthma and } \\
\text { gonorrhea } \\
(38),(39)\end{array}$ & $\begin{array}{l}\text { Antimutagenicity } \\
\text { properties (40) }\end{array}$ & Tree \\
\hline MSM19/4 & Leguminosae & Acacia nilotica (L.) Delile & Lkiloriti & Bark & $\begin{array}{l}\text { Hot bark } \\
\text { decoction } \\
\text { treats } \\
\text { indigestion, } \\
\text { pneumonia, } \\
\text { stomachache, } \\
\text { painful joints, } \\
\text { gonorrhea, } \\
\text { and coughs }\end{array}$ & $\begin{array}{l}\text { Treat } \\
\text { stomachache } \\
\text { after birth } \\
(17,21), \text { skin } \\
\text { wounds (41) } \\
\text { retained } \\
\text { placenta (42) }\end{array}$ & $\begin{array}{l}\text { Presence of } \\
\text { tannins, } \\
\text { saponins, } \\
\text { flavonoids (43) }\end{array}$ & Tree \\
\hline
\end{tabular}




\begin{tabular}{|c|c|c|c|c|c|c|c|c|}
\hline $\begin{array}{l}\text { Voucher } \\
\text { Number }\end{array}$ & Family & Latin name & Local name & $\begin{array}{l}\text { Part } \\
\text { used }\end{array}$ & Treatment & $\begin{array}{l}\text { Documented } \\
\text { traditional } \\
\text { uses }\end{array}$ & $\begin{array}{l}\text { Reported } \\
\text { pharmacological } \\
\text { activity }\end{array}$ & Habit \\
\hline MSM19/14 & & $\begin{array}{l}\text { Acacia oerfota (Forssk.) } \\
\text { Schweinf. }\end{array}$ & Ldepe & Bark & $\begin{array}{l}\text { Bark } \\
\text { decoction } \\
\text { treats cough, } \\
\text { gonorrhoea, } \\
\text { stomachache } \\
\text { and jaundice }\end{array}$ & $\begin{array}{l}\text { STDs, } \\
\text { postpartum } \\
\text { tonic, } \\
\text { facilitate } \\
\text { lactation, } \\
\text { rejuvenation } \\
\text { (5) athristis } \\
\text { and STDs } \\
\text { (42) }\end{array}$ & $\begin{array}{l}\text { Antifungal } \\
\text { activity from } \\
\text { Root bark } \\
\text { extracts (44) }\end{array}$ & Shrub \\
\hline MSM19/5 & & Entada leptostachya Harms & Ldalampoi & Bark & $\begin{array}{l}\text { Root } \\
\text { decoction } \\
\text { treats bone } \\
\text { diseases, } \\
\text { gout }\end{array}$ & $\begin{array}{l}\text { Tuberculosis } \\
\text { and cough } \\
\text { (35), boils } \\
\text { and } \\
\text { abscesses } \\
(45)\end{array}$ & $\begin{array}{l}\text { barks had the } \\
\text { highest } \\
\text { antioxidant } \\
\text { activity (46) }\end{array}$ & Climb \\
\hline MSM20/18 & Lythraceae & Lawsonia inermis $\mathrm{L}$. & Lgiriai & Root & $\begin{array}{l}\text { Root } \\
\text { decoction to } \\
\text { treat } \\
\text { stomachache, } \\
\text { gallstones, } \\
\text { induce } \\
\text { diarrhea }\end{array}$ & $\begin{array}{l}\text { Treats } \\
\text { fibroids (47), } \\
\text { Elephantiasis } \\
(42)\end{array}$ & $\begin{array}{l}\text { Antioxidant } \\
\text { activity (48) }\end{array}$ & Shrub \\
\hline MSM19/3 & Primulaceae & Myrsine africana $\mathrm{L}$. & Seketet & Seeds & $\begin{array}{l}\text { Decoction of } \\
\text { ground seeds } \\
\text { for } \\
\text { deworming, } \\
\text { stomach } \\
\text { pains, } \\
\text { common } \\
\text { colds }\end{array}$ & $\begin{array}{l}\text { Bone and } \\
\text { joint pain, } \\
\text { kidney } \\
\text { disease, } \\
\text { gonorrhea, } \\
\text { women's } \\
\text { health (49), } \\
\text { STDs (32) }\end{array}$ & $\begin{array}{l}\text { Antihelmintic } \\
\text { efficacy (28) }\end{array}$ & Shrub \\
\hline MSM19/2 & Rhamnaceae & Rhamnus prinoides $\mathrm{L}$. & Lkinyil & Root & $\begin{array}{l}\text { Root } \\
\text { decoction } \\
\text { treats } \\
\text { common } \\
\text { cold, fever, } \\
\text { pneumonia, } \\
\text { Malaria }\end{array}$ & $\begin{array}{l}\text { STDs, back } \\
\text { and joint } \\
\text { aches, } \\
\text { arthritis, aids } \\
\text { in digestion, } \\
\text { tonic }(5,50)\end{array}$ & $\begin{array}{l}\text { Antimalarial } \\
\text { activity (51) }\end{array}$ & Shrub \\
\hline MSM19/8 & & Rhamnus staddo A.Rich. & Lkukulai & $\begin{array}{l}\text { Root, } \\
\text { Bark }\end{array}$ & $\begin{array}{l}\text { Root } \\
\text { decoction } \\
\text { treats } \\
\text { malaria, fever, } \\
\text { common } \\
\text { colds, cleans } \\
\text { the blood }\end{array}$ & $\begin{array}{l}\text { STDs, } \\
\text { flu/cold (52), }\end{array}$ & $\begin{array}{l}\text { antiplasmodial } \\
\text { activities (53) }\end{array}$ & Shrub \\
\hline MSM19/12 & Rutaceae & Toddalia asiatica (L.) Lam. & Leparmunyo & Root & $\begin{array}{l}\text { Root } \\
\text { decoction } \\
\text { used for } \\
\text { common } \\
\text { cold, } \\
\text { pneumonia } \\
\text { and malaria } \\
\text { especially in } \\
\text { children }\end{array}$ & $\begin{array}{l}\text { Treats } \\
\text { malaria (6) }\end{array}$ & $\begin{array}{l}\text { Potential } \\
\text { antidiabetic and } \\
\text { antioxidant } \\
\text { properties to } \\
\text { treat diabetes } \\
\text { (54) shows } \\
\text { antinflammaoty } \\
\text { activities (55) }\end{array}$ & Climb \\
\hline MSM19/11 & & Zanthoxylum chalybeum Engl & Loisuki & Seeds & $\begin{array}{l}\text { Hot decoction } \\
\text { treats throat } \\
\text { problems and } \\
\text { tonsils }\end{array}$ & $\begin{array}{l}\text { Treatment of } \\
\text { malaria (56) }\end{array}$ & $\begin{array}{l}\text { Antiplasmodial } \\
\text { activity from the } \\
\text { leaves (57) }\end{array}$ & Shrub \\
\hline MSM19/13 & Salvadoraceae & Salvadora Persica L. & Sokotei & $\begin{array}{l}\text { Root, } \\
\text { Bark }\end{array}$ & $\begin{array}{l}\text { Root } \\
\text { decoction to } \\
\text { treat stomach } \\
\text { pain, a paste } \\
\text { is made to } \\
\text { treat } \\
\text { toothache }\end{array}$ & $\begin{array}{l}\text { Eye } \\
\text { infections, } \\
\text { worms, } \\
\text { malaria, } \\
\text { stomach } \\
\text { ache, } \\
\text { constipation, } \\
\text { tonic, cold, } \\
\text { teeth hygiene, } \\
\text { respiratory } \\
\text { infections (5), }\end{array}$ & $\begin{array}{l}\text { Extract } \\
\text { possesses dose } \\
\text { dependent anti- } \\
\text { inflammatory } \\
\text { (58), } \\
\text { antimicrobial } \\
\text { agent (59) }\end{array}$ & Shrub \\
\hline $\begin{array}{l}\text { Voucher } \\
\text { Number }\end{array}$ & Family & Latin name & Local name & $\begin{array}{l}\text { Part } \\
\text { used }\end{array}$ & Treatment & $\begin{array}{l}\text { Documented } \\
\text { traditional } \\
\text { uses }\end{array}$ & $\begin{array}{l}\text { Reported } \\
\text { pharmacological } \\
\text { activity }\end{array}$ & Habit \\
\hline
\end{tabular}




\begin{tabular}{|c|c|c|c|c|c|c|c|c|}
\hline $\begin{array}{l}\text { Voucher } \\
\text { Number }\end{array}$ & Family & Latin name & Local name & $\begin{array}{l}\text { Part } \\
\text { used }\end{array}$ & Treatment & $\begin{array}{l}\text { Documented } \\
\text { traditional } \\
\text { uses }\end{array}$ & $\begin{array}{l}\text { Reported } \\
\text { pharmacological } \\
\text { activity }\end{array}$ & Habit \\
\hline MSM19/7 & Santalaceae & $\begin{array}{l}\text { Osyris lanceolata Hochst. \& } \\
\text { Steud. }\end{array}$ & Losiesia & Bark & $\begin{array}{l}\text { Bark } \\
\text { decoction } \\
\text { cleans } \\
\text { stomach after } \\
\text { childbirth, } \\
\text { stops } \\
\text { diarrhoea in } \\
\text { children }\end{array}$ & $\begin{array}{l}\text { Treats cancer } \\
(60) \text {, asthma } \\
\text { and sore } \\
\text { throat (32) }\end{array}$ & $\begin{array}{l}\text { Antioxidants } \\
\text { properties } \\
(61,62)\end{array}$ & Tree \\
\hline MSM20/19 & Urticaceae & Urtica massaica Mildbr. & Sapai & Leaves & $\begin{array}{l}\text { Boiled and } \\
\text { the decoction } \\
\text { is used to } \\
\text { treat } \\
\text { stomachache }\end{array}$ & $\begin{array}{l}\text { Stomachache } \\
\text { (5) }\end{array}$ & $\begin{array}{l}\text { Antimicrobial } \\
\text { activities (63) }\end{array}$ & Herb \\
\hline MSM20/20 & Vitaceae & Cissus rotundifolia Vahl & Raraiti & Root & $\begin{array}{l}\text { Root } \\
\text { decoction } \\
\text { treats chest } \\
\text { problems }\end{array}$ & $\begin{array}{l}\text { Stomachache } \\
\text { (20) }\end{array}$ & $\begin{array}{l}\text { Anti- } \\
\text { inflammatory } \\
\text { potency (64) }\end{array}$ & Climb \\
\hline MSM20/23 & & Cissus quadrangulari $\mathrm{L}$ & Sukurtuti & $\begin{array}{l}\text { Root, } \\
\text { Leaves }\end{array}$ & $\begin{array}{l}\text { Boiled roots } \\
\text { treat chest } \\
\text { pains. } \\
\text { Crushed } \\
\text { leaves given } \\
\text { to sick calves }\end{array}$ & $\begin{array}{l}\text { Upper } \\
\text { respiratory } \\
\text { problems (65) }\end{array}$ & $\begin{array}{l}\text { Antibacterial } \\
\text { activity (66), } \\
\text { antibacterial and } \\
\text { antimicrobial } \\
\text { activity (67) }\end{array}$ & Climb \\
\hline MSM20/17 & & & Lolpiyayo & $\begin{array}{l}\text { Root, } \\
\text { Bark }\end{array}$ & $\begin{array}{l}\text { It is crushed } \\
\text { into a powder } \\
\text { and sniffed to } \\
\text { cure } \\
\text { headaches. }\end{array}$ & $\begin{array}{l}\text { Malaria and } \\
\text { colds }(65)\end{array}$ & & N/A \\
\hline
\end{tabular}

STDs = Sexually transmitted diseases, KFR = Kirisia Forest Reserve

$\mathrm{Y}-\mathrm{Yes}, \mathrm{N}-\mathrm{No}$

Table 2

Medicinal plants collected from Kirisia Forest Reserve

\begin{tabular}{|llll|}
\hline Voucher Number & Family & Latin name & Local name \\
\hline MSM20/24 & Anacardiaceae & Rhus natalensis & Lmisigiyoi \\
\hline MSM19/10 & Apocynaceae & Carissa spinarum L. & Lamuria \\
\hline MSM20/21 & Canellaceae & Warburgia ugandensis Sprague & Sokoni \\
\hline MSM19/6 & Ebenaceae & Euclea divinorum Hiern & Lchingei \\
\hline MSM20/22 & & Eurphoria candelabrum & Lakirding'ai \\
\hline MSM19/9 & Lamiaceae & Ajuga integrifolia Buch.-Ham & Mpopong'i \\
\hline MSM19/1 & & Rotheca myricoides (Hochst.) Steane \& Mabb. & Lmakutikuti \\
\hline MSM19/3 & Primulaceae & Myrsine africana L. & Seketet \\
\hline MSM19/2 & Rhamnaceae & Rhamnus prinoides L. & Lkinyil \\
\hline MSM19/8 & & Rhamnus staddo A.Rich. & Lkukulai \\
\hline MSM19/12 & Rutaceae & Toddalia asiatica (L.) Lam. & Leparmunyo \\
\hline MSM19/11 & & Zanthoxylum chalybeum Engl & Loisuki \\
\hline MSM19/7 & Santalaceae & Osyris lanceolata Hochst. \& Steud. & Losiesia \\
MSM20/19 & Urticaceae & Urtica massaica Mildbr. & Sapai \\
\hline
\end{tabular}

Multiple plant parts either single or mixed together were the most sold plant parts at $29 \%$ followed by roots and barks at $25 \%$ and leaves at $9 \%$ respectively. Data from the growth habit of the traded plants shows that, shrubs were the highest with 11 species (48\%), followed by trees 5 ( $26 \%)$ climbers 4 (17\%) and herbs 2 (9\%) as shown in (Fig. 2).

The family with most species was Leguminosae (3), Lamiaceae (2), Rhamnaceae (2), and Rutaceae (2) other families were represented by one species. The plants are prepared by first boiling them and consumed as either a cold or hot decoction mixed with either tea, soup or milk. Mostly administered orally apart from "Lolpiyayo" which is sniffed after crushing the dried bark into a powder. 


\section{Discussion}

Based on the market surveys, the use of medicinal plants to manage and treat different ailments is a sought-after option in Maralal. The traders sold their products every day in the open market, they have a designated area donated by the County government of Samburu. None of the traders had any formal certification or license to trade. All the twenty-four traded plants are native to Kenya. Only one plant "Lolpiyayo" could not be identified by neither its English name nor its scientific name. Five of the traded species are among the most popular and commercially important medicinal plants in sub-Saharan African; Acacia nilotica (L.) Delile, C. spinarum, R. prinoides, T. asiatica and S. Persica (68). Compared to other studies in Kenya, the number of traded plants in Maralal was relatively low $(3,49,69)$. Some of the traded plants were similar to those traded in Marsabit such as Carissa spinarum $\mathrm{L}$, Croton dichogamus Pax, Myrsine africana L. and Rotheca myricoides (Hochst.) Steane \& Mabb. (49). Another five species recorded from our market surveys have previously been recorded in a similar study done in two urban cities A. integrifolia, C. spinarum, M. africana L, R. prinoides, and Warburgia ugandensis Sprague (3). "Lekeek isiet" the cold decoction made from eight plants species was used to treat Malaria and Pneumonia. Seven of the eight species used to make "Lekeek isiet" were among those traded in the market apart from C. megalocarpus. Roots and barks were the most traded parts similar to Marsabit and Moyale (49) due to their long shelf life (70) while leaves were the least used parts. The majority of the traded plants were used to treat malaria, common coughs, stomachaches/pains, women's health problems, and STIs. It was noted that $R$. myricodes was one of the strongest and most dangerous plants that only a specialist can administer because it can lead to death if wrongly used. Most of the traders prescribed the medicinal plants to customers based on symptoms of the illness. The traders did not collect the plants themselves, they had collectors who supplied them with the products. It was noted that plant species traded depended on their supply and availability from the wild. This is supported by the fact that during the first market survey (August 2019), only fifteen species were available in the market. During the second survey (January 2020), nine additional species were available while four species from the first survey were not available in the market. This indicates that it is likely that more than twenty-four plants are traded based on supply and availability considering some species are collected as far as shrublands in Baragoi (102 Kms) and Wamba (107 Kms) from Maralal town. None of the traded plants is listed under IUCN as endangered; however, the traders stated that some of the plants were becoming rare to find mostly due to deforestation, anthropogenic activities (19) and drought. Overharvesting was noted as the major factor contributing to the decline especially for plants with high demand and where roots and barks were harvested. This was the case with $O$. lanceolate which the traders stated that it had become rare to find both in the shrublands and in Kirisia forest Reserve. Apart from its medicinal qualities, the species is exploited for its essential oils used in the perfume industry. The popularity and increase in demand for the $R$. myricodes and $M$. africana threaten populations in the wild with the species becoming scarce hence the need for sustainable extraction and management. Increase in market demand and overharvesting of medicinal plants from the wild threatens with loss of biodiversity $(49,71)$. Local extinction of species such as Olea europaea L. and Rhamnus prinoides L. have been reported in Loitoktok (69). The traders stated that the knowledge was orally passed down generations; however, the majority of the traders lacked apprentices because the younger generation opted for alternative livelihoods in urban cities. This leads to the loss of traditional knowledge on medicinal plants because the mastery is not passed down or shared. Additionally, the use of traditional knowledge on medicinal plants use faces threats from the allopathic healthcare sector because the former is not formally recognized in Kenya (69). Since the trade is informal, it is difficult to get any figures on estimates of how much plant product is traded in term of quantity for any given period. None of the interviewed traders kept any records of their sales.

\section{Conclusion}

This study highlights the importance of medicinal plants as the basis of traditional medicine. A total of 24 species from 16 families were recorded from the market surveys. Trade of medicinal plants is an important source of income for many both in the rural and urban areas in Kenya. Unfortunately, most of the trade in Kenyan markets is undocumented hence little is known on the conservation status of most traded species. Therefore, there is a need to develop a complete database of medicinal plant species that are used in Kenya. This will guide in conservation efforts especially for overharvested species such as $O$. lanceolate, R. myricoides, and M. africana. Assessing the current population status of important medicinal plants in the wild will aid in the establishment of conservation measures as well as sustainable exploitation. Since the trade is informal, it was difficult to get any figures on how much plant product is traded.

\section{Declarations}

\section{Acknowledgments}

I'm grateful to all the participants especially the traders who shared their knowledge. I also want to thank the translator and research assistant who enabled a smooth process during data collection during market surveys and in the wild. This study is part of a Ph.D. research project at Karatina University, Kenya.

\section{Ethics approval and consent to participate}

Permission was granted by the local authorities and the people interviewed before undertaking the study.

\section{Consent for publication}

The people interviewed were made aware that the information collected could be made public.

\section{Competing interests}

The author declares no competing interests.

\section{Availability of data and materials}


The datasets used during the current study are available from the corresponding author on request.

\section{Funding}

Self-funded

\section{Author details}

School of Science and Applied Technology,

Department of Earth Sciences, Laikipia University,

P.O. Box 1100-20300, Nyahururu, Kenya.

\section{References}

1. Kigen GK, Ronoh HK, Kipkore WK, Rotich JK. Current trends of Traditional Herbal Medicine Practice in Kenya: A review. J Pharmacol Ther. 2013;2(1):32-7.

2. World Health Organization (WHO). WHO Traditional Medicine Strategy 2002-2005. World Heal Organ Geneva [Internet]. 2002;1-74. Available from: http://www.wpro.who.int/health_technology/book_who_traditional_medicine_strategy_2002_2005.pdf.

3. Njoroge GN. Traditional medicinal plants in two urban areas in Kenya (Thika and Nairobi): Diversity of traded species and conservation concerns. Ethnobot Res Appl. 2012;10:329-38.

4. Nagata JM, Jew AR, Kimeu JM, Salmen CR, Bukusi EA, Cohen CR. Medical pluralism on Mfangano Island: Use of medicinal plants among persons living with HIV/AIDS in Suba District, Kenya. J Ethnopharmacol. 2011.

5. Kimondo J, Miaron J, Mutai P, Njogu P. Ethnobotanical survey of food and medicinal plants of the llkisonko Maasai community in Kenya. J Ethnopharmacol [Internet]. 2015;175(October):463-9. Available from: http://dx.doi.org/10.1016/j.jep.2015.10.013.

6. Nankaya J, Nampushi J, Petenya S, Balslev H. Ethnomedicinal plants of the Loita Maasai of Kenya. Environ Dev Sustain [Internet]. 2020;22(3):2569-89. Available from: https://doi.org/10.1007/s10668-019-00311-w.

7. Ticktin T. The ecological implications of harvesting non-timber forest products. J Appl Ecol. 2004;41(1):11-21.

8. Quiroz D, Towns A, Legba SI, Swier J, Brière S, Sosef M, et al. Quantifying the domestic market in herbal medicine in Benin, West Africa. J Ethnopharmacol. 2014;151(3):1100-8.

9. Jusu A, Sanchez AC. Medicinal Plant Trade in Sierra Leone: Threats and Opportunities for Conservation. Econ Bot. 2014;68(1):16-29.

10. Cuni-Sanchez A, Pfeifer M, Marchant R, Burgess ND. Ethnic and locational differences in ecosystem service values: Insights from the communities in forest islands in the desert. Ecosyst Serv [Internet]. 2016;19:42-50. Available from: http://dx.doi.org/10.1016/j.ecoser.2016.04.004.

11. Łuczaj $Ł$, Lamxay V, Tongchan K, Xayphakatsa K, Phimmakong K, Radavanh S, et al. Wild food plants and fungi sold in the markets of Luang Prabang, Lao PDR. J Ethnobiol Ethnomed. 2021;17(1).

12. Van Andel T, Behari-Ramdas J, Havinga R, Groenendijk S. The medicinal plant trade in Suriname. Ethnobot Res Appl. 2007;5:351-72.

13. Otieno J, Abihudi S, Veldman S, Nahashon M, van Andel T, de Boer HJ. Vernacular dominance in folk taxonomy: A case study of ethnospecies in medicinal plant trade in Tanzania. J Ethnobiol Ethnomed. 2015;11(1):1-7.

14. Van Andel T, Myren B, Van Onselen S. Ghana's herbal market. J Ethnopharmacol [Internet]. 2012;140(2):368-78. Available from: http://dx.doi.org/10.1016/j.jep.2012.01.028.

15. National Coordinating Agency for Population \& Development. Seeking Solutions for Traditional Herbal Medicine: Kenya Develops a National Policy. Policy Br No 1. (Brief No. 1):1-4.

16. KNBS. 2019 Kenya population and housing census Volume IV. Vol. 2, 2019 Kenya population and housing census. 2019.251 p.

17. Fratkin E. Traditional Medicine and Concepts of Healing Among Samburu Pastoralists of Kenya. J Ethnobiol. 1996;16(1):63-97.

18. Bussmann RW. Islands in the Desert-Forest Vegetation of Kenya'S Smaller Mountains and Highland Areas (Nyiru, Ndoto, Kulal, Marsabit, Loroghi, Ndare, Mukogodo, Porror, Mathews, Gakoe, Imenti, Ngaia, Nyambeni, Loita, Nguruman, Nairobi). J East African Nat Hist [Internet]. 2002 Jan 1;91(1):27-79. Available from: https://doi.org/10.2982/0012-8317(2002)91[27:IITDVO]2.0.CO.

19. Nanyingi MO, Mbaria JM, Lanyasunya AL, Wagate CG, Koros KB, Kaburia HF, et al. Ethnopharmacological survey of Samburu district, Kenya. J Ethnobiol Ethnomed. 2008;4:1-12.

20. Omwenga E, Mbugua P, Okemo P. Ethno-Medicinal Survey of Important Plants of Samburu Community (Wamba)-Samburu District in Kenya. Res Rev J Bot Sci. 2014;3(3):25-9.

21. Spencer P. Samburu Notions of Health and Disease, and their Relationship to Inner Cleanliness. African Conceptions Heal Dis [Internet]. 1959; Available from: http://eprints.soas.ac.uk/8764/2/Samburu_notions_of_health.pdf.

22. Hitimana J, Ole Kiyiapi JL, Wambui P, Kisioh H, Mayienda R, Warinwa F, et al. Challenges of Linking Socio-Economic Significance and Conservation Value of Forests in Drylands of Kenya: Case Study of Kirisia Forest-Samburu Pastoralists Coexistence. Biol Divers Sustain Resour Use. 2011;(June 2014).

23. IT K, JM M, PK G. M M, D KJ, SG K. Cytotoxicity of selected medicinal plants extracts using the brine shrimp lethality assay from Samburu county, Kenya. J Med Res. 2018;4(5):249-55.

24. Njoroge PW, Opiyo SA. Some Antibacterial and Antifungal Compounds from Root Bark of Rhus natalensis. Am J Chem. 2019;9(5):150-8. 
25. Jeruto P, Lukhoba C, Ouma G, Otieno D, Mutai C. An ethnobotanical study of medicinal plants used by the Nandi people in Kenya. J Ethnopharmacol. 2008;116(2):370-6.

26. MUNDARAGI A, THANGADURAI D. Process optimization, physicochemical characterization and antioxidant potential of novel wine from an underutilized fruit Carissa spinarum L. (Apocynaceae). Food Sci Technol [Internet]. 2018;38:428-33. Available from: http://www.scielo.br/scielo.php? script=sci_arttext\&pid=S0101-20612018000300428\&nrm=iso.

27. Olila D, Olwa-Odyek, Opuda-Asibo J. Antibacterial and antifungal activities of extracts of Zanthoxylum chalybeum and Warburgia ugandensis, Ugandan medicinal plants. Afr Health Sci [Internet]. 2001 Dec;1(2):66-72. Available from: https://pubmed.ncbi.nlm.nih.gov/12789119.

28. Gathuma JM, Mbaria JM, Wanyama J, Kaburia HFA, Mpoke L, Mwangi JN. Efficacy of Myrsine africana, Albizia anthelmintica and Hilderbrantia sepalosa herbal remedies against mixed natural sheep helminthosis in Samburu district, Kenya. J Ethnopharmacol. 2004;91(1):7-12.

29. Kipkore W, Wanjohi B, Rono H, Kigen G. A study of the medicinal plants used by the Marakwet Community in Kenya. J Ethnobiol Ethnomed [Internet]. 2014;10(1):1-22. Available from: Journal of Ethnobiology and Ethnomedicine.

30. Al-Fatimi M. Antifungal Activity of Euclea divinorum Root and Study of its Ethnobotany and Phytopharmacology. Processes. 2019 Oct 1;7:680.

31. Mwonjoria M. Antinociceptive effects of dichloromethane extract of Euclea divinorum Lin. 2018 Nov 28;7:1104-7.

32. Hilonga S, Otieno JN, Ghorbani A, Pereus D, Kocyan A, de Boer H. Trade of wild-harvested medicinal plant species in local markets of Tanzania and its implications for conservation. South African J Bot [Internet]. 2019;122:214-24. Available from: https://doi.org/10.1016/j.sajb.2018.08.012.

33. 33. et al. Botanical description, ethnomedicinal uses, phytochemistry and pharmacological effects of Croton dichogamus Pax (Euphorbiaceae). $J$ Phytopharm. 2021;10(1):42-7.

34. Shehu M, Bello I, Abdulkardir N, Shehu A, Jamil SE, Waziri SA. Utilization of medicinal plants used in the management of HIV/AIDS opportunistic infections in njeru sub-county, buikwe district, Uganda. MOJ Bioequivalence Bioavailab. 2018;5(1):66-72.

35. Wagate CG, Mbaria JM, Gakuya DW, Nanyingi MO, Kareru PG, Njuguna A, et al. Screening of some Kenyan medicinal plants for antibacterial activity. Phyther Res [Internet]. 2010;24(1):150-3. Available from: http://dx.doi.org/10.1002/ptr.2866.

36. Asnake S, Teklehaymanot T, Hymete A, Erko B, Giday M. Evaluation of the antiplasmodial properties of selected plants in southern Ethiopia. BMC Complement Altern Med [Internet]. 2015;15(1):448. Available from: https://doi.org/10.1186/s12906-015-0976-x.

37. Cocquyt K, Cos P, Herdewijn P, Maes L, Van den Steen PE, Laekeman G. Ajuga remota Benth.: From ethnopharmacology to phytomedical perspective in the treatment of malaria. Phytomedicine [Internet]. 2011;18(14):1229-37. Available from: http://www.sciencedirect.com/science/article/pii/S0944711311003308.

38. Hayelom K, Mekbeb A, Eyasu M, Wondwossen E, Kelbesa U. Methanolic effect of clerodendrum myricoides root extract on blood, liver and kidney tissues of mice. Afr Health Sci. 2012;12(4):489-97.

39. Mukungu N, Abuga K, Okalebo F, Ingwela R, Mwangi J. Medicinal plants used for management of malaria among the Luhya community of Kakamega East sub-County, Kenya. J Ethnopharmacol [Internet]. 2016;194:98-107. Available from: http://dx.doi.org/10.1016/j.jep.2016.08.050.

40. Verschaeve L, Van Staden J. Mutagenic and antimutagenic properties of extracts from South African traditional medicinal plants. J Ethnopharmacol [Internet]. 2008;119(3):575-87. Available from: http://www.sciencedirect.com/science/article/pii/S037887410800305X.

41. Dalle G, Maass B, Isselstein J. Plant Biodiversity and Ethnobotany of Borana Pastoralists in Southern Oromia, Ethiopia. Econ Bot. 2005 Mar;1:59:43-65.

42. Teklehaymanot T. An ethnobotanical survey of medicinal and edible plants of Yalo Woreda in Afar regional state, Ethiopia. J Ethnobiol Ethnomed. 2017;13(1).

43. Auwal MS, Saka S, Mairiga IA, Sanda KA, Shuaibu A, Ibrahim A. Preliminary phytochemical and elemental analysis of aqueous and fractionated pod extracts of Acacia nilotica (Thorn mimosa). Vet Res forum an Int Q J [Internet]. 2014;5(2):95-100. Available from: https://pubmed.ncbi.nlm.nih.gov/25568701.

44. Elfadil H, Fahal A, Kloezen W, Ahmed EM, van de Sande W. The In Vitro Antifungal Activity of Sudanese Medicinal Plants against Madurella mycetomatis, the Eumycetoma Major Causative Agent. PLoS Negl Trop Dis [Internet]. 2015 Mar 13;9(3):e0003488. Available from: https://doi.org/10.1371/journal.pntd.0003488

45. Kisangau DP, Herrmann TM. Utilization and conservation of medicinal plants used for primary health care in Makueni district, Kenya. Int J Biodivers Sci Manag. 2007;3(3):184-92.

46. Ruto M, Ngugi C, Kareru P, Kipyegon C, Sylvester R, Madivoli E, et al. Antioxidant activity and antimicrobial properties of Entada leptostachya and Prosopis juliflora extracts. J Med Plants Econ Dev. 2018 Feb 27;2:1-8.

47. Kaingu CK, Oduma JA, Mbaria JM, Kiama SG. Medicinal plants traditionally used for the management of female reproductive health dysfunction in Tana River County, Kenya. Tang [Humanitas Med. 2013;3(2):17.1-10.

48. Badoni Semwal R, Semwal DK, Combrinck S, Cartwright-Jones C, Viljoen A. Lawsonia inermis L (henna): Ethnobotanical, phytochemical and pharmacological aspects. J Ethnopharmacol [Internet]. 2014;155(1):80-103. Available from:

http://www.sciencedirect.com/science/article/pii/S0378874114004115.

49. Delbanco AS, Burgess ND, Cuni-Sanchez A. Medicinal Plant Trade in Northern Kenya: Economic Importance, Uses, and Origin1. Econ Bot. 2017;71(1):1331.

50. Muthee JK, Gakuya DW, Mbaria JM, Kareru PG, Mulei CM, Njonge FK. Ethnobotanical study of anthelmintic and other medicinal plants traditionally used in Loitoktok district of Kenya. J Ethnopharmacol [Internet]. 2011;135(1):15-21. Available from: http://dx.doi.org/10.1016/j.jep.2011.02.005.

51. Muregi F, Ishih A, Suzuki T, Kino H, Amano T, Mkoji G, et al. In Vivo antimalarial activity of aqueous extracts from Kenyan medicinal plants and their Chloroquine (CQ) potentiation effects against a blood-induced CQ-resistant rodent parasite in mice. Phytother Res. 2007 Apr 1;21:337-43.

Page $9 / 12$ 
52. Kiringe JW. A Survey of Traditional Health Remedies Used by the Maasai of Southern Kaijiado District, Kenya. Ethnobot Res Appl. $2006 ; 4: 061$.

53. Muregi FW, Ishih A, Miyase T, Suzuki T, Kino H, Amano T, et al. Antimalarial activity of methanolic extracts from plants used in Kenyan ethnomedicine and their interactions with chloroquine (CQ) against a CQ-tolerant rodent parasite, in mice. J Ethnopharmacol [Internet]. 2007;111(1):190-5. Available from: http://www.sciencedirect.com/science/article/pii/S0378874106005927.

54. Stephen Irudayaraj S, Sunil C, Duraipandiyan V, Ignacimuthu S. Antidiabetic and antioxidant activities of Toddalia asiatica (L.) Lam. leaves in Streptozotocin induced diabetic rats. J Ethnopharmacol [Internet]. 2012;143(2):515-23. Available from: http://dx.doi.org/10.1016/j.jep.2012.07.006.

55. Kariuki HN, Kanui TI, Yenesew A, Patel N, Mbugua PM. Antinocieptive and anti-inflammatory effects of toddalia asiatica (L) Lam. (Rutaceae) root extract in Swiss albino mice. Pan Afr Med J. 2013;14(L):3-7.

56. Bbosa G. Antiplasmodial Activity of Leaf Extracts of Zanthoxylum chalybeum Engl. Br J Pharm Res. 2014;4(6):705-13.

57. Waiganjo B, Moriasi G, Onyancha J, Elias N, Muregi F Antiplasmodial and Cytotoxic Activities of Extracts of Selected Medicinal Plants Used to Treat Malaria in Embu County, Kenya. Marchand B, editor. J Parasitol Res [Internet]. 2020;2020:8871375. Available from:

https://doi.org/10.1155/2020/8871375.

58. Ahmad M, Imran H, Yaqeen Z, Rehman Z, Rahman A, Fatima N, et al. Pharmacological profile of Salvadora Persica. Pak J Pharm Sci. 2011 Jul;1:24:32330.

59. Abhary M, Al-Hazmi A-A. Antibacterial activity of Miswak (Salvadora persica L.) extracts on oral hygiene. J Taibah Univ Sci [Internet]. 2016;10(4):513-20. Available from: http://dx.doi.org/10.1016/j.jtusci.2015.09.007.

60. Chhabra SC, Mahunnahb RLA, Mshiub EN. 199 1) 143-157) and hTraditional Medicine Research Unit. J Ethnopharmacol. 1991;33:143-57.

61. Yeboah EMO, Majinda RRT. Radical scavenging activity and total phenolic content of extracts of the root bark of Osyris lanceolata. Nat Prod Commun. 2009;4(1):89-94.

62. Ochanga O, Kilonzo M. Antioxidant properties of aqueous and ethyl acetate extracts of some plants used as herbal tea in Tanzania. Oxid Antioxid Med Sci. 2018;7(2):1.

63. Allan K, Lizzy M, Christine BII, Brian K. The antimicrobial activity of the leaves of Urtica massaica on Staphylococcus aureus. Escherichia coli. 2019;7(2):21-4.

64. Sen MK, Dash BK. A review on phytochemical and pharmacological aspects of Cissus quadrangularis L. Int J Green Pharm. 2012;6(3):169-73.

65. Grillo KM. The materiality of mobile pastoralism: ethnoarchaeological perspectives from Samburu, Kenya. Azania Archaeol Res Africa. 2012;47(4):540-1.

66. Shah U. Cissus quadrangularis L. Phytochemicals, traditional uses and pharmacological activities - A review. Int J Pharm Pharm Sci. 2011;3(SUPPL. 4):41-4.

67. Kaur R, Malik CP. Cissus quadrangularis L - Its botany, chemistry and medicinal importance: A review. Int J Pharm Clin Res. 2014;6(1):27-35.

68. Van Wyk BE. A review of commercially important African medicinal plants. J Ethnopharmacol [Internet]. 2015;176:118-34. Available from: http://dx.doi.org/10.1016/j.jep.2015.10.031.

69. Ngaruiya GW. Reweaving stakeholder networks: Promoting climate mitigation and Maasai culture using medicinal plants in Kenya. Ecosyst Serv. 2015.

70. van Andel TR, Croft S, van Loon EE, Quiroz D, Towns AM, Raes N. Prioritizing West African medicinal plants for conservation and sustainable extraction studies based on market surveys and species distribution models. Biol Conserv. 2015.

71. Moyo M, Aremu AO, Van Staden J. Medicinal plants: An invaluable, dwindling resource in sub-Saharan Africa. J Ethnopharmacol. 2015.

\section{Figures}




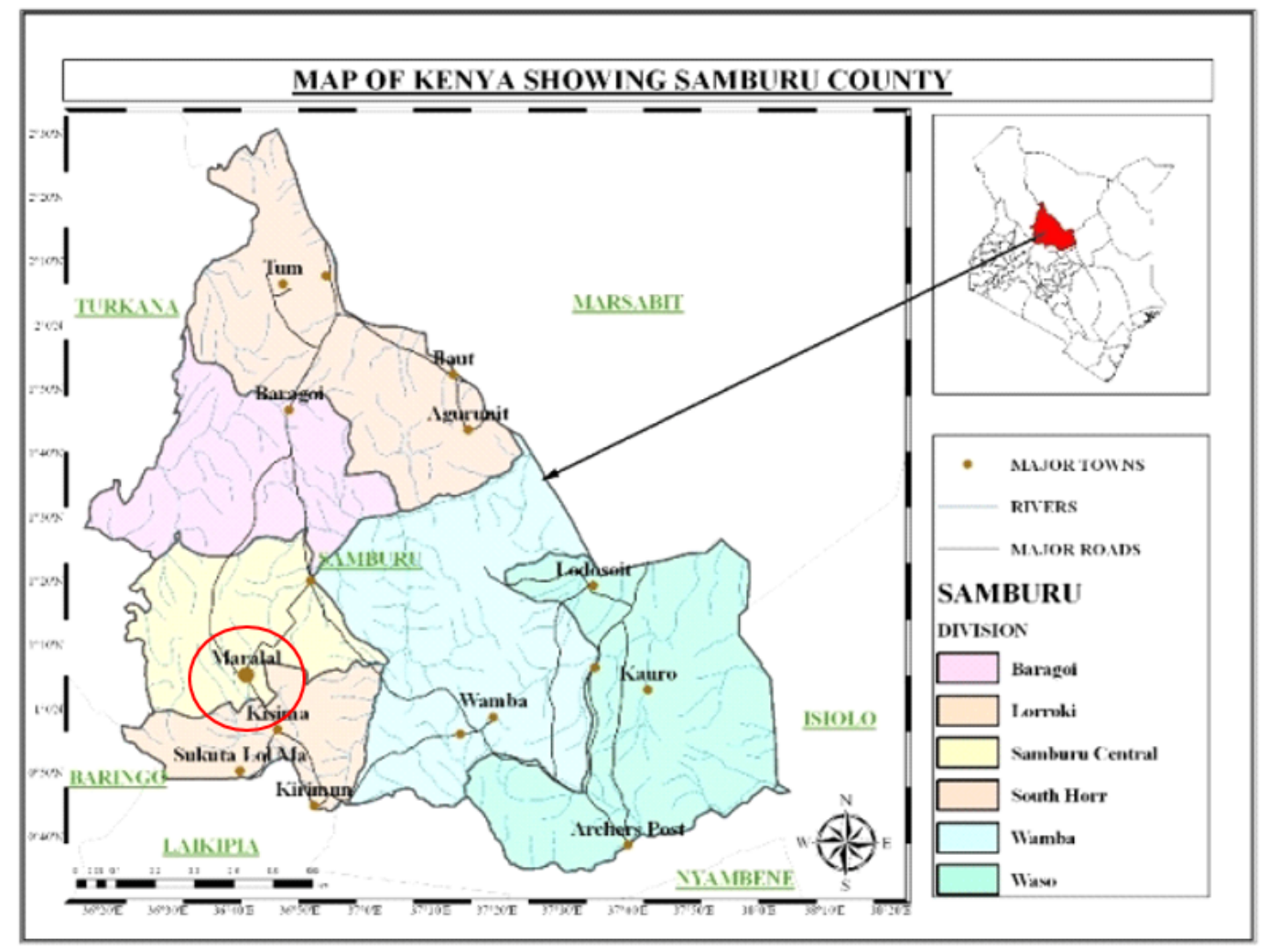

\section{Figure 1}

Map of a study area in Samburu County (23) Note: The designations employed and the presentation of the material on this map do not imply the expression of any opinion whatsoever on the part of Research Square concerning the legal status of any country, territory, city or area or of its authorities, or concerning the delimitation of its frontiers or boundaries. This map has been provided by the authors.
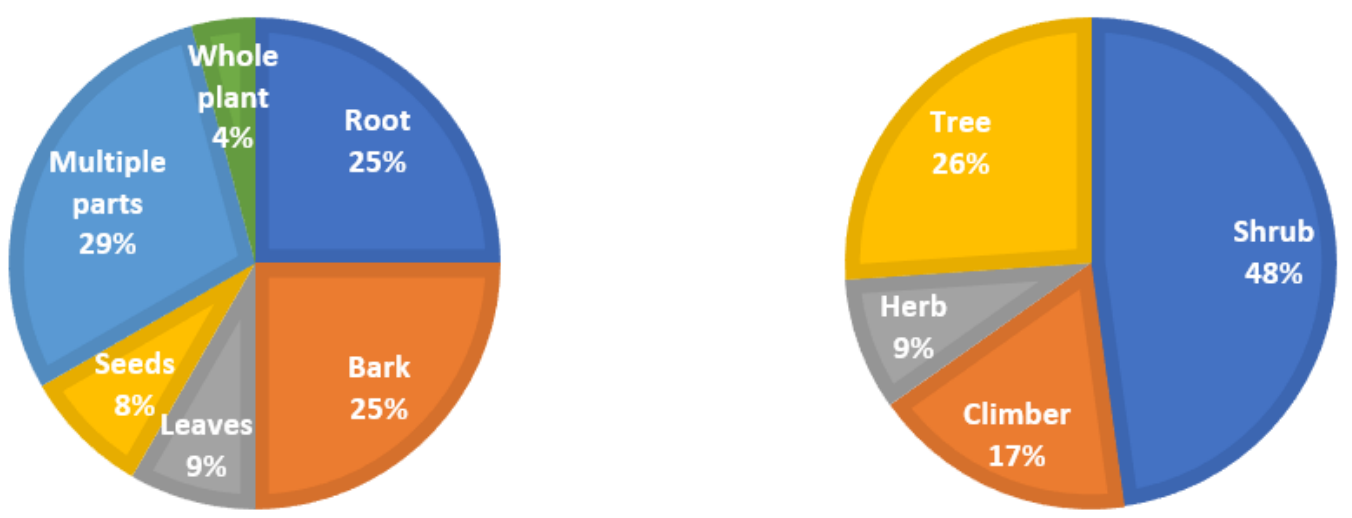

Figure 2

Plant parts traded (left) and growth habit (right)

\section{Supplementary Files}

This is a list of supplementary files associated with this preprint. Click to download.

- p1.png

- p2.png

- p3.png 
- p4.png

- p5.png

- p6.png

Page $12 / 12$ 